\title{
Enhancing polycystic ovarian syndrome awareness using private social network
}

\author{
Mohammed Alotaibi ${ }^{1}$, Amani Ali Shaman ${ }^{2}$ \\ ${ }^{1}$ Faculty of Computers and Information Technology, ${ }^{2}$ Faculty of Medicine, University of Tabuk, Tabuk, Saudi Arabia \\ Contributions: (I) Conception and design: M Alotaibi; (II) Administrative support: All authors; (III) Provision of study materials or patients: AA \\ Shaman; (IV) Collection and assembly of data: All authors; (V) Data analysis and interpretation: All authors; (VI) Manuscript writing: All authors; (VII) \\ Final approval of manuscript: All authors. \\ Correspondence to: Dr. Mohammed Alotaib. Faculty of Computers and Information Technology, University of Tabuk, Tabuk, Saudi Arabia. \\ Email: mmalotaibi@ut.edu.sa.
}

Background: The purpose of the study is to evaluate the effectiveness of mobile health technology such as private social network (PCOS system) in creating awareness about the disease management among the women diagnosed with the polycystic ovarian syndrome.

Methods: A pilot study was conducted in Tabuk, Saudi Arabia. The study was carried out for a period of three months using a private social network as an intervention. The private social network is used as an interface to educate and create awareness among the participants in the study. Fifty women diagnosed with PCOS were randomly selected and were distributed in to control group (doesn't use PCOS system) and intervention group (use the private social network). The level of the polycystic ovarian syndrome awareness was measured using a structured questionnaire at the beginning and end of the follow-up period.

Results: More than 80 posts and 10 pictures contain data about PCOS were conveyed by medical staff. Also, about 120 private SMS messages between medical staff and the patients were refined. The after effects of this investigation demonstrated that the intervention had better awareness about polycystic ovary syndrome management compared to the control group $(\mathrm{F}=18.25 ; \mathrm{P}<0.0001)$.

Conclusions: This study of the private social network among the PCOS patients demonstrated that mobile health technology such as the private social network can substantially enhance the PCOS disease awareness levels among Saudi women. The PCOS System can also help in reducing the costs as most of the disease management process is handled over the application through the messages and feedbacks is provided through the application, and in saving time by avoiding hospital visits. This study is first-of-its kind conducted in Saudi Arabia.

Keywords: Polycystic ovarian syndrome; mobile health; social network

Received: 02 February 2019; Accepted: 20 November 2019; Published: 05 October 2020.

doi: 10.21037/mhealth.2019.12.02

View this article at: http://dx.doi.org/10.21037/mhealth.2019.12.02

\section{Introduction}

Polycystic ovarian syndrome refers to an endocrine condition that actually is entailing the myriad symptoms that helps promulgate from a wide range of different hormonal disturbances. Furthermore, it is considered as the common endocrine disorder that can lead to infertility in women during their reproductive ages (1). The primary driver of the disease remains to be a mystery; however, considerable evidence shows that the there is a likelihood of metabolic and also reproductive abnormalities that change the hormonal distress, which includes high oestrogen and androgen levels in the females $(2,3)$. Thus, the hormonal distress associated with the loss of ordinary hormonal input prompts high $\mathrm{LH}$ and low progesterone relentless state.

Besides, polycystic ovarian syndrome is commonly 
observed in the adolescent stages, and it's frequently described by the dysfunction of the ovarian tube and hyperandrogenism. Prominently, this condition is dangerous to women and has a higher predominance of causing some major complexities that interfere with their health and the quality of life (4). The most widely recognized symptoms are attributed to the production of an overabundance of androgen that prompts anovulation, skin inflammation, and hirsutism. Techniques like ultrasound examination for polycystic ovarian morphology display, anovulation, and overabundance of androgen using clinical or biochemical tests are used in diagnosing PCOS (5). According to the Rotterdam criteria, it is critical for health specialists to guarantee that at least two out of three benchmarks which include (I) oligo- or anovulation, (II) clinical and/or biochemical signs of hyperandrogenism, or (III) polycystic ovaries; have been met in order to confirm the PCOS.

Moreover, the PCOS can lead to different physical complexities among women like skin inflammation, baldness, obesity, hirsutism and hair loss. Thus, women suffering PCOS undergo metabolic changes identified with dyslipidemia, diabetes type 2 and also the resistance from the insulin. In addition, women diagnosed with PCOS are also susceptible from experiencing the different psychological problems like anxiety, depression and stress (6). It is also important to understand that women diagnosed with PCOS also face different complications and symptoms that obstruct the normal working of various gynaecological frameworks such as menses disorder, infertility, ovulation issues, and endometrial cancer.

It is essential for the health specialists to recognize the impact of the disease on their mental and physical states and also in the social environment. In addition, the health specialists must take up the responsibility to create awareness about the effects and complications of PCOS among the diagnosed women, so that the women can take necessary actions to manage the disease effectively, minimizing the chance of complications that may arise; thereby improving the quality of life. Every year around $25 \%$ to $30 \%$ of women aged 30 years old, diagnosed with PCOS display weakened glucose intolerance, and about $8 \%$ are prone to other side effects like type 2 diabetes (5). Moreover, women experiencing PCOS are prone to endometrial, ovarian, and breast cancer because of the unopposed estrogen secretion that causes the anovulation (6).

Various studies (7-10) conducted on raising awareness levels of the PCOS patients in effectively managing the disease have shown positive results in mitigating the psychological aspects like overcoming the depression, stress and additionally empowering the patients to understand the complexities of the disease, and the ways to overcome such complexities. The patients need to know how the disease can be managed by practicing healthy lifestyles like making physical exercise (11-13) as a part of their daily routine, maintaining healthy diet. This process can help them in developing the positive attitude towards the disease management. However, the there are no programs developed in the aspect of increasing awareness about disease management and the practices of increasing public awareness levels about PCOS management in Saudi Arabia are ineffective or missing (11). They suggested that the medical specialists in the Saudi Arabia must take steps to increase the awareness about PCOS. Prior studies have shown that PCOS patients when provided with sufficient data about the disease, and a secure platform to connect and share their experiences through technology platforms like mobile applications can enhance their quality of the life, self-esteem, and confidence in managing the disease $(14,15)$. However, there is a lack of research on elaborating how the health specialists in Saudi Arabia are creating awareness about PCOS management; and there is an urgent need to promote research studies in this area to investigate the possible use of mobile health technologies which have shown positive results in managing other similar diseases (16). The occurrence of PCOS at young ages could lead to stress among the patients and might also affect their health and lifestyles as the disease is associated with major complications. Therefore, it is essential and necessary to raise awareness about the PCOS and the self-management practices among the PCOS patients (11).

The use of mobile health technologies is increasing rapidly for effective delivery of healthcare services and also for managing the chronic diseases like diabetes, PCOS etc., which require various self-management practices after the diagnosis. There are various studies that have suggested the positive impact of using mobile health technologies in creating awareness for effective management of chronic diseases $(16,17)$.

The number of mobile phone and internet technologies users in the kingdom of Saudi Arabia is increasing rapidly in the recent years. Such changes in the use of technology by the people can make it easier to implement mobile health systems for effective management of chronic diseases and the delivery of healthcare remotely, which can result in reduced costs and improved efficiency in the healthcare operations. Studies $(16,18)$ have indicated that the prevalence of PCOS 


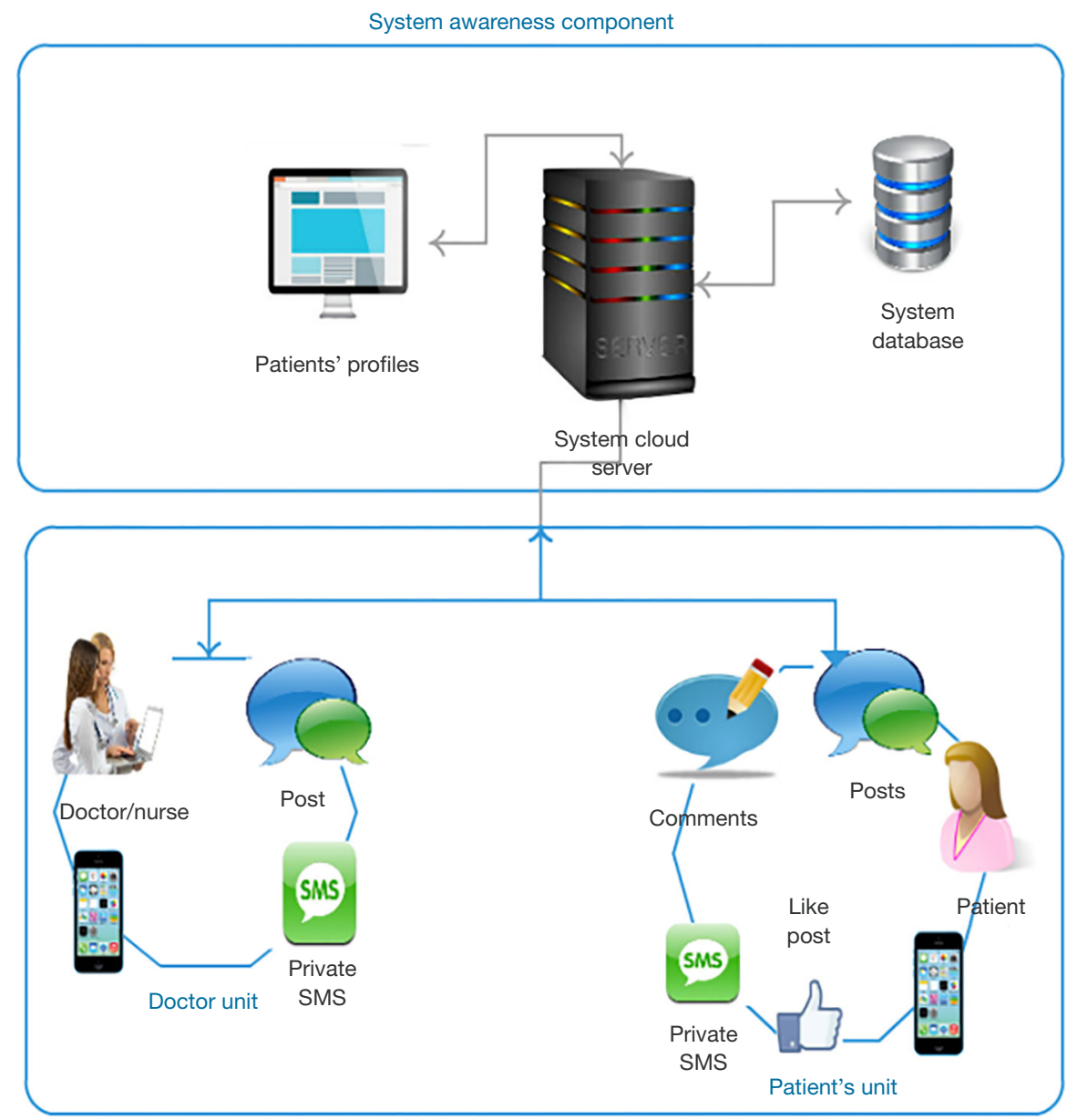

Mobile patient/healthcare provider component

Figure 1 System architecture. Source: (19).

and its related diseases are increasing and it was observed that there was no clinical study that was conducted on analyzing the effectiveness of self-managing PCOS through an integrated mobile application and text messaging system in Saudi Arabia. Considering the above discussed factors, it was assessed that there is a need to create awareness about PCOS management among the people in order to reduce the complications that could arise after the disease is diagnosed.

Therefore, this study focused on evaluating the effectiveness of mobile health technology by using an integrated PCOS system with mobile application (application SnapScreen) and SMS in creating awareness about PCOS among the patients.

\section{The PCOS mobile system}

This paper presents the design of PCOS mobile system that can be used for creating awareness about effective management of PCOS and also the impact and effectiveness of the system in creating the awareness among the PCOS patients in Saudi Arabia. The paper also presents the complete system's engineering along with the data acquisition process (19). Figure 1 demonstrates the PCOS framework that which is divided in to two parts.

The system as shown in the Figure 1 is divided in to two major components including disease awareness component and the private social network component. The disease awareness component includes the cloud server which also used for administration and for providing disease related information. The private social network component is divided in to three classes including doctor's unit, patient's unit and admin unit, from which the doctors and patients can access the information through the mobile application. 


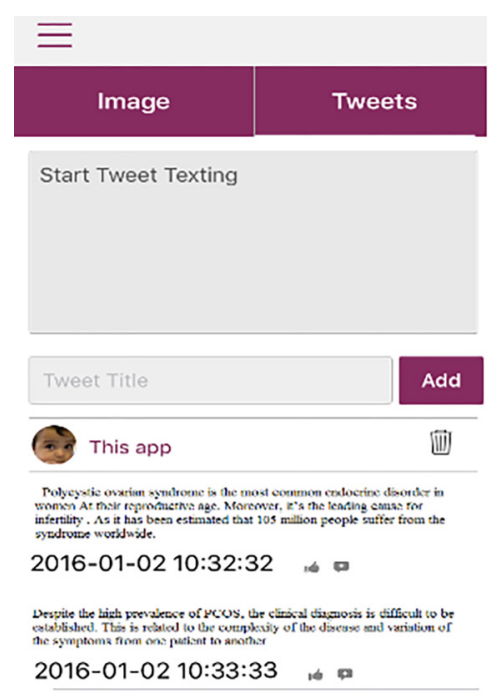

Figure 2 Srnap screens of the mobile App.

The system is modelled according to the Saudi Arabian healthcare system. The components of the system are explained in the following sections. A sample of the mobile app screen is shown in Figure 2 above and more in the supplementary file.

\section{Diseases awareness component}

This component mainly deals with the back-end operations which include the creation of patient's profiles, loading lab reports, adding and sending disease related awareness information, and doctor's profiles. The patients and doctors access information from the cloud server used in the component. The database uses MySQL (20) for database operations, and is monitored by database administrator who monitors and keeps track of patient's information and vital records.

\section{Private social network components}

It is in general a social network like Twitter with some changes to fit our requirements like the number of post's characters. We extend the message to be 250 characters rather than 140 in Twitter. One of most challenge when using common social network like Twitter OR Facebook is the users' admirations as the Twitter or Facebook admin who can validate users and insure that person is really a physician or no. We usually see some people present

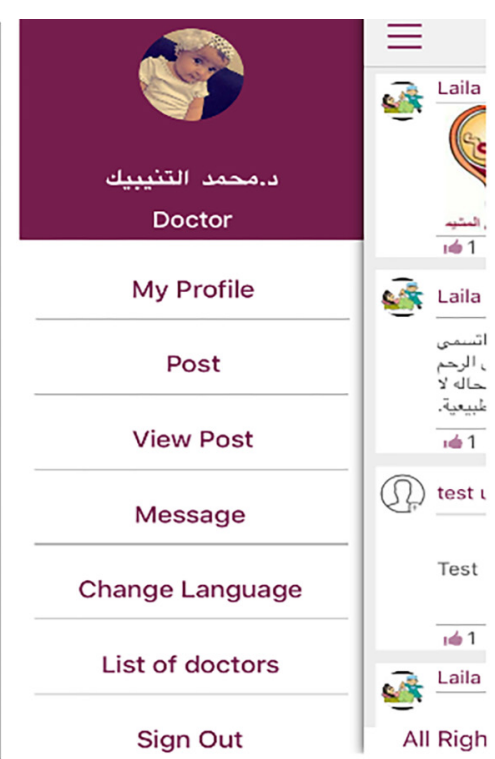

themselves as physician without the icon of validation on their accounts. It is difficult to trust what they are tweeting. In this private social network, we can validate, block, delete and edit physician's profile based on their proof of ID to insure they are really physician.

Furthermore, this component is designed for accessing the information by the end users, and acts as a segment that ensures cooperation between three units of the system including patient's unit; doctor/nurse unit; and the administrator unit.

\section{Patient's unit}

This unit includes the PCOS patients, and it is mandatory for them to have smartphones/mobiles to access their health data, awareness information about PCOS, management techniques, and to make any enquires about the disease if they wish to. Using a mobile application, the patients can also like and comment on any post that is posted by the specialists. Additionally, they can also share their experience by posting or by sharing a picture, which can be viewed by other patients. Other patients can also react to the post by liking or commenting on the experience posted by the patients. To address the problem of misuse the medical staff or administrator can erase any objectionable posts or content. The patients can also ask any information that may be required relating to a post or information posted by the medical staff, which will be addressed by the medical staff through a reply. The easy-to-use interface of the mobile 
application provides an effective way to communicate by the patients. The mobile application is embedded with private SMS messaging features for making private communication with their doctors and friends at the application, changing profile pictures, following other individuals.

\section{Doctor/nurse unit}

The mobile application provides an effective graphical user interface (GUI) which would enable the doctors and nurses in easily accessing the information about a patient, diagnosis process, and help the patients in increasing their knowledge and awareness about PCOS by involving in the social network with their patient and guide the $\mathrm{m}$ indirect way. The private messaging feature allows the doctors and nurses to respond individually to the patients and safe the patient's privacy. The medical staff can also evaluate any posting by the patients and may delete it if they consider it to be objectionable or misleading.

\section{The admin unit}

All the technical aspects of the system are overseen by the admin staff at the hospital location. They monitor the functioning of the system, and repair or update the system whenever it is required. In addition, they can also upgrade the system with the changing needs, and add or remove functionalities as authorised by the responsible authorities. The admin has full access on all contents of the system and users' profiles.

\section{Methods}

\section{Study setting \& participants}

A randomised controlled trial (RCT) was conducted in order to evaluate the effectiveness of the PCOS system in creating awareness about the disease management of PCOS. The RCT study was conducted over a period of 3 months (March-June 2017) in the city of Tabuk, Saudi Arabia. The research study was approved by King Salman with reference R\&REC2016-116. The participants were recruited during their hospital visits. The participants during their visit are explained with the purpose of the study and an informed consent was made as a necessary step to recruit the participants. Fifty-four women participants diagnosed with PCOS, belonging to the age group 20-40 were selected for the study. Participants who gave their consent to participate in the study were randomly divided in to two groups including the intervention group [22] and the control group [32].
The participants in both the groups were asked to take a survey (pre-study) before taking part in the study which contain multiple choice questionnaire (11). The study used SPSS 2.0. for the analysis of the pre and post study survey results. The flowchart of the study is presented in the Figure 3.

\section{Intervention group}

The participants in the intervention group were given training for using the PCOS mobile application. To provide more flexibility and ease-of-use, the application was developed in the Saudi Arabia native language Arabic. The general functions associated with the intervention group are explained in the following points.

* The medical staff will be providing information about PCOS and its management techniques on a daily basis which can be accessed by the patients through the mobile application $24 \times 7$;

* The participants in the intervention group have access to special services like sending an enquiry through private messaging to the medical staff. Additionally, they can also post, comment and share awareness related messages;

* The medical staff can review the posts and comments made by the participants and remove them if they consider them to be objectionable or misleading.

\section{Control group}

The participants in the control group did not receive any mobile application based help, and their awareness levels are increased by traditional methods like explaining them during their hospital visits and receiving advices through awareness programs conducted in local and primary healthcare centres.

\section{Data analysis}

The statistical package for social sciences SPSS version 2.0. (SPSS Inc., Chicago, IL, USA) was used for analyzing the data collected in pre and post study surveys. The data was properly described and analyzed by comparing the results between the control and intervention groups before and after the intervention study.

\section{Results}

Fifty-four participants diagnosed with polycystic ovary syndrome who fulfilled all inclusion criteria were enrolled 


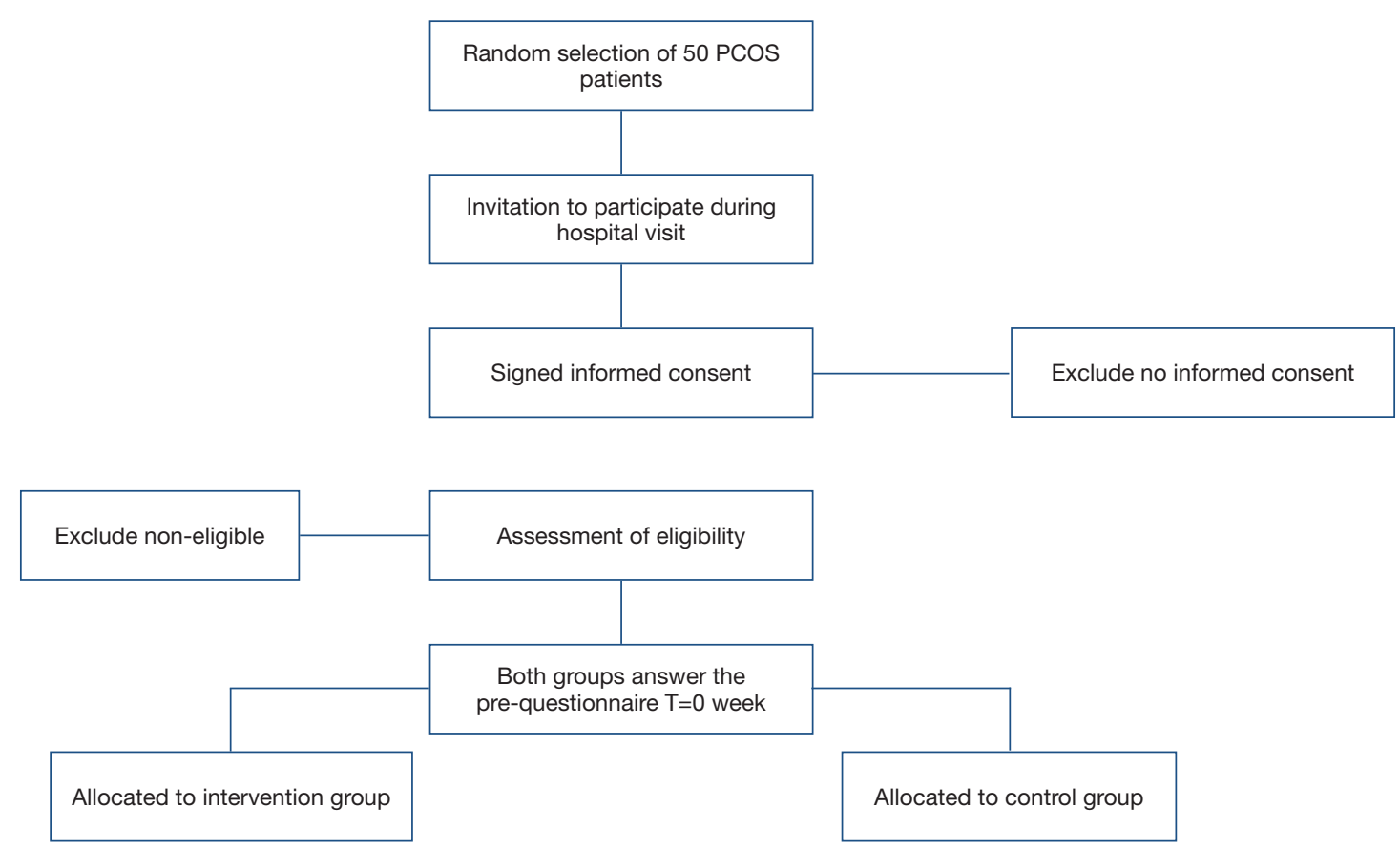

Figure 3 Pilot study plan.

for the study. Twenty-two (40.74\%) participants were randomly assigned to the intervention group, and the remaining 32 participants were assigned to the control group. The PCOS awareness levels of the participants were tested using the online survey questionnaire available on google forms before and after the intervention study. The intervention group received the training and awareness information through a dedicated mobile application, while the control group did not receive any awareness training through mobile application. Participants' information including age, city, education levels, weight and stature was collected at the gauge. Whenever enlisted, the respondents in the two gatherings were comparable in qualities concerning the benchmark attributes as shown in Table 1 .

\section{Outcome of intervention study}

In the baseline, a pre-study online questionnaire survey was carried out to assess the initial awareness levels of the participants, which is then proceeded by 3 months of follow-up. At the end of 3-month study, the same online questionnaire survey was conducted to assess the change in the awareness levels about PCOS among the participants in the both groups. Thirty participants (55.56\%) completed the study, out of which $17(77.27 \%)$ are from the intervention group and 13 (40.63\%) from the control group.

General linear model utilizing rehashed estimation investigation was used to evaluate the findings collected from both intervention and control groups. The outcome demonstrated that there was significant change in additional time among the participants in intervention group compared with the participants in control group $(\mathrm{F}=18.25$; $\mathrm{P}<0.0001)$.

At benchmark the participants from control group were found to be having more awareness about polycystic ovary disorder compared to the intervention group (mean \pm SD $=13.69 \pm 4.46$ in control group compared with $10.88 \pm 6.15$ in intervention group). However, after the intervention study, the awareness levels of the participants in the intervention group were increased to a greater extent compared to that of the control group $(14.71 \pm 6.24$ compared with $14.77 \pm 3.75$ ), as shown in the Table 2. The level of awareness about polycystic ovary syndrome for those with mobile application intervention enhanced better $(\mathrm{F}=5.90 ; \mathrm{P}=0.005)$ compared with those who didn't receive any intervention.

The results of the intervention and control groups plotted against evaluated minimal mean are presented in Figure 4, which reflects the change in the awareness levels among the groups before and after the intervention study. 
Table 1 Baseline characteristics of the participant enrolled in intervention and control arms

\begin{tabular}{lcc}
\hline Factor & Intervention, $\mathrm{n}(\%)$ & Control, $\mathrm{n}(\%)$ \\
\hline Number of respondents & $22(40.74)$ & $32(59.26)$ \\
Age (mean \pm SD) & $26.73 \pm 5$ & $29.34 \pm 4.84$ \\
City (Tabuk) & $22(100.0)$ & $30(93.75)$ \\
Education & & $1(3.13)$ \\
Illiterate & $0.0(0.0)$ & $3(9.38)$ \\
Primary & $2(9.10)$ & $7(21.88)$ \\
Secondary & $3(13.60)$ & $5(15.62)$ \\
High school & $7(31.8)$ & $16(50.0)$ \\
University & $10(45.5)$ & $69.53 \pm 16.06$ \\
Weight (mean \pm SD) & $74.57 \pm 14.71$ & $153.19 \pm 19.36$ \\
\hline
\end{tabular}

Table 2 Evaluation of effectiveness of intervention regarding polycystic ovary syndrome

\begin{tabular}{lcccc}
\hline Groups & Before & After & F & P value \\
\hline Intervention & $10.88 \pm 6.15$ & $14.71 \pm 6.24$ & 18.252 & $<0.001$ \\
Control & $13.69 \pm 4.46$ & $14.77 \pm 3.75$ & & \\
\hline
\end{tabular}

\section{Discussion}

Polycystic ovarian syndrome is a common endocrine disorder-affecting the women in their reproductive ages (21). PCOS can lead to various complications among women, including reproductive (infertility, miscarriage and pregnancy complications), metabolic (obesity, insulin resistance, pre diabetes, type 2 diabetes $\mathrm{T} 2 \mathrm{DM})$, and Psychological (anxiety, depression) health aspects (22). The considerations of complications and the measures to raise awareness to for effective management of PCOS were lacking in the recent studies. In a study (11) on awareness about signs, symptoms, complications and self-management approaches of PCOS were lacking in more than $60 \%$ of the motivated sample population.

Women with PCOS need to rely on information to manage and limit its impact on their health and lifestyle (23). Source of information can include advice from physicians; books; PCOS support group; and information available on Internet. Various studies have indicated that women with PCOS did not have positive experience with their doctors regarding initial information about the disease given by the doctor. Furthermore, some women were not satisfied with information provided by Physician, and felt that they were more informed about PCOS than the doctors (17). Internet is considered as the best source to obtain information about the disease, as it ensures privacy, sense of control, and upto-date information at their fingertips (17). However, the major limitation in this aspect is that there is no control over quality and credibility of information exchanged online (24).

In this paper, a PCOS system integrated with mobile application and SMS technology was presented for PCOS patients in KSA. The design aspects of the system were clearly outlined and explained with the use and functionality of each component and the unit. The performance of the system and its effectiveness in increasing the awareness among the patients is evaluated using a pilot study in the city of Tabuk, Saudi Arabia.

The outcomes demonstrated the huge change in the PCOS awareness levels of the intervention group compared to that of the control group. The outcomes are in concurrence with other worldwide investigations that demonstrate the effectiveness of advanced mobile phone intervention of chronic malady like diabetes (16). These findings show that PCOS system's framework and approach 


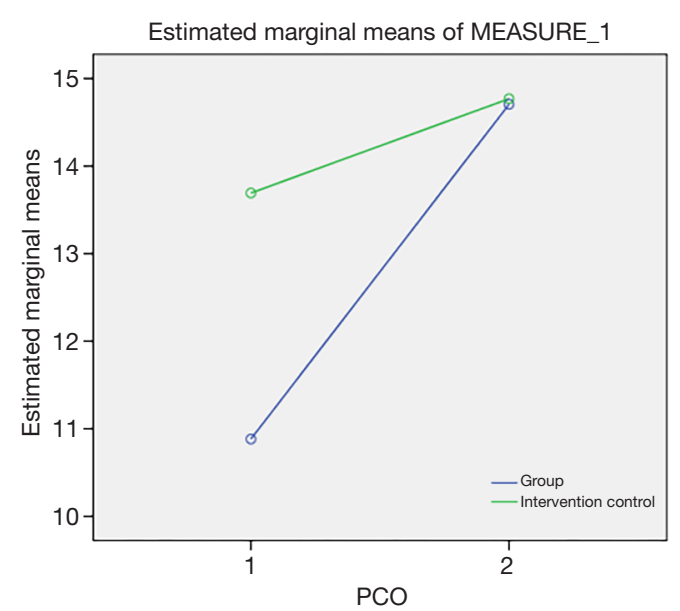

Figure 4 Comparison between intervention and control group before and after intervention regarding polycystic ovary syndrome.

can be an effective solution in enhancing the PCOS awareness among the Saudi patients.

The effective PCOS system proposed in this study can help the patients in the process of learning by providing access to pertinent data about PCOS management. This process can be very effective and useful in the rural areas of Saudi Arabia, where there is limited access to healthcare services, as it ensures remote delivery of healthcare services and disease management.

The PCOS system thus can help in delivering the healthcare services remotely thereby reducing the burden of healthcare costs and improving the efficiency of the healthcare delivery and services. The study also had few limitations. A small sample population of 54 patients was used in the study, and in addition nearly $40 \%$ of the participants did not complete the study. As a result, the findings can be related in specific to the population in a particular region (Tabuk).

The current social conventions that may oppose in the selection of new advanced technologies, for example, portable mobile health solutions related arrangements are needed to be addressed in the country. These difficulties can be mitigated by adopting fundamental measures, for example, (I) adopting an efficient and effective m-health system for conveying pressing answer for most restorative difficulties, for instance, PCOS predominance as a significant aspect of the national e-medicine services design in the nation; (II) increasing the portable health innovations by enhancing the awareness among the therapeutic group and their therapeutic preparing; (III) enhance and extend the framework for versatile advances that can better encourage the mobile health solutions arrangements in the social insurance division; (IV) presence of $\mathrm{m}$-health as a component of the medical educational modules; (V) the process of expressing freely in gaining knowledge, anticipates versatile health among PCOS patients in the Kingdom.

\section{Conclusions}

In future investigation, we will include new capacity which is compressed in dealing with the PCOS and remotely screen the PCOS persistence as presented in the framework portrayed in (19). The future work will integrate other functionalities like booking hospital appointments for PCOS patients, a notification based chart scheduling daily activities.

\section{Acknowledgments}

Funding: None.

\section{Footnote}

Conflicts of Interest: Both authors have completed the ICMJE uniform disclosure form (available at http://dx.doi. org/10.21037/mhealth.2019.12.02). The authors have no conflicts of interest to declare.

Ethical Statement: The authors are accountable for all aspects of the work in ensuring that questions related to the accuracy or integrity of any part of the work are appropriately investigated and resolved. The study was conducted in accordance with the Declaration of Helsinki (as revised in 2013). The research study was approved by King Salman with reference R\&REC2016-116. Informed consent was taken from all subjects.

Open Access Statement: This is an Open Access article distributed in accordance with the Creative Commons Attribution-NonCommercial-NoDerivs 4.0 International License (CC BY-NC-ND 4.0), which permits the noncommercial replication and distribution of the article with the strict proviso that no changes or edits are made and the original work is properly cited (including links to both the formal publication through the relevant DOI and the license). See: https://creativecommons.org/licenses/by-nc-nd/4.0/. 


\section{References}

1. Sedighi S, Amir Ali Akbari S, et al. Comparison of lifestyle in women with polycystic ovary syndrome and healthy women. Glob J Health Sci 2014;7:228-34.

2. Guraya SS. Prevalence and ultrasound features of polycystic ovaries in young unmarried Saudi females. J Microsc Ultrastruct 2013;1:30-4.

3. El Hayek S, Bitar L, Hamdar LH, Mirza FG, Daoud G. Poly Cystic Ovarian Syndrome: An Updated Overview. Front Physiol 2016;7:124.

4. Thornton EC, Von Wald T, Hansen K. Polycystic Ovarian Syndrome: A Primer. S D Med 2015;68:257-61.

5. Broekmans FJ, Knauff EA, Valkenburg O, et al. PCOS according to the Rotterdam consensus criteria: Change in prevalence among WHO-II anovulation and association with metabolic factors. BJOG 2006;113:1210-7.

6. Moran L, Gibson-Helm M, Teede H, et al. Polycystic ovary syndrome: a biopsychosocial understanding in young women to improve knowledge and treatment options. J Psychosom Obstet Gynaecol 2010;31:24-31.

7. Tomlinson J, Letherby G, Pinkney J, et al. Raising awareness of polycystic ovary syndrome. Nurs Stand 2013;27:35-9.

8. Gul S, Zahid A, Ansari A. PCOS: Symptoms and Awareness in Urban Pakistani Women. Available online: https:// www.researchgate.net/publication/271707254_PCOS_ Symptoms_and_Awareness_in_Urban_Pakistani_Women

9. Pitchai P, Sreeraj SR, Anil PR. Awareness of lifestyle modification in females diagnosed with polycystic ovarian syndrome in India: explorative study. Int J Reprod Contracept Obstet Gynecol 2016;5:470-6.

10. Sunanda B, Sabitha N. A Study to Assess the Knowledge Regarding PCOS (Polycystic Ovarian Syndrome) among Nursing Students at NUINS. Available online: http:// nitte.edu.in/journal/september2016/02.pdf

11. AlSinan A, Shaman AA. A Study to Measure the Health Awareness of Polycystic Ovarian Syndrome in Saudi Arabia. Glob J Health Sci 2017;9:130.

12. Nybacka $\AA$, Carlström K, Ståhle A, et al. Randomized comparison of the influence of dietary management and/ or physical exercise on ovarian function and metabolic parameters in overweight women with polycystic ovary syndrome. Fertil Steril 2011;96:1508-13.

13. Jedel E, Labrie F, Odén A, et al. Impact of electroacupuncture and physical exercise on hyperandrogenism and oligo/amenorrhea in women with polycystic ovary syndrome: a randomized controlled trial. Am J Physiol Endocrinol Metab 2011;300:E37-45.

14. Stankiewicz M, Norman R. Diagnosis and management of polycystic ovary syndrome: a practical guide. Drugs 2006;66:903-12.

15. Memish ZA, El Bcheraoui C, Tuffaha M, et al. Obesity and associated factors--Kingdom of Saudi Arabia, 2013. Prev Chronic Dis 2014;11:E174.

16. Alotaibi MM, Istepanian R, Philip N. A mobile diabetes management and educational system for type-2 diabetics in Saudi Arabia (SAED). Mhealth 2016;2:33.

17. Avery JC, Braunack-Mayer AJ. The information needs of women diagnosed with Polycystic Ovarian Syndrome-implications for treatment and health outcomes. BMC Womens Health 2007;7:9.

18. Guraya SS. Prevalence and ultrasound features of polycystic ovaries in young unmarried Saudi females. J Microsc Ultrastruct 2013;1:30-4.

19. A mobile Polycystic ovarian syndrome management and awareness system for Gulf countries: System architecture. Available online: https://ieeexplore.ieee.org/ document/7556124

20. MySQL 5.7 Reference Manual. Available online: https:// dev.mysql.com/doc/refman/5.7/en/

21. Naderpoor N, Shorakae S, de Courten B, et al. Metformin and lifestyle modification in polycystic ovary syndrome: systematic review and meta-analysis. Hum Reprod Update 2015;21:560-74.

22. Teede H, Deeks A, Moran L. Polycystic ovary syndrome: a complex condition with psychological, reproductive and metabolic manifestations that impacts on health across the lifespan. BMC Med 2010;8:41.

23. Lobo RA. Priorities in polycystic ovary syndrome. Med J Aust 2001;174:554-5.

24. Holbrey S, Coulson NS. A qualitative investigation of the impact of peer to peer online support for women living with polycystic ovary syndrome. BMC Womens Health 2013;13:51.

doi: $10.21037 /$ mhealth.2019.12.02

Cite this article as: Alotaibi M, Shaman AA. Enhancing polycystic ovarian syndrome awareness using private social network. mHealth 2020;6:33. 


\section{Application SnapScreen}

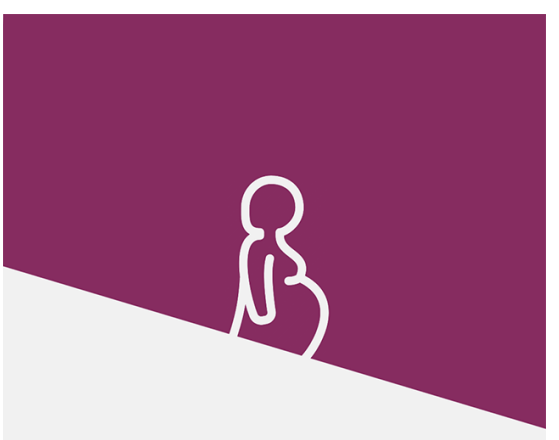

Polycystic Ovarian Syndrome

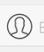

8

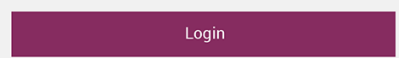

Forget Passward? Don't have an account? Sign Up

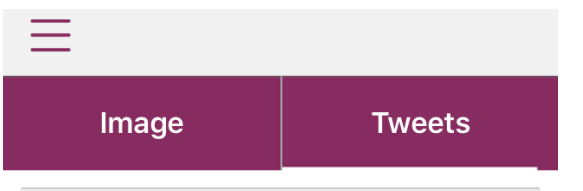

Start Tweet Texting

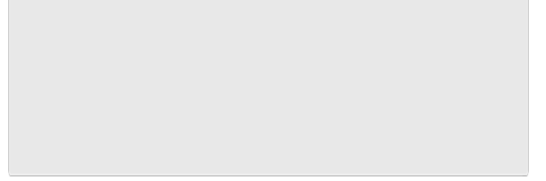

Tweet Title

Add

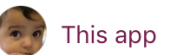

||111

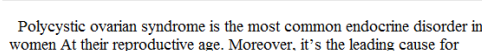

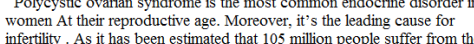
infertility. A i it has be
syndrome worldwide.

2016-01-02 10:32:32

Despite the high prevalence of PCOS, the clinical diagnosis is difificult to be
established. This is related to the complexity of the disease and variation of

2016-01-02 10:33:33
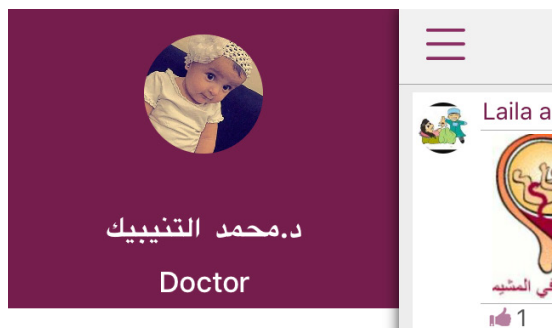

at 1

.

Laila a

My Profile

Post

\begin{tabular}{c}
\hline View Post \\
\hline Message \\
\hline Change Language \\
\hline List of doctors \\
\hline Sign Out
\end{tabular}

\section{Questionaire}

Name:

Age:

City:

Educational level:

\begin{tabular}{|c|c|c|c|}
\hline & Yes & No & I don't know \\
\hline \multicolumn{4}{|l|}{ Follicle is a small fluid filled sac with a single egg inside and the follicles are often called cysts } \\
\hline \multicolumn{4}{|l|}{ Polycystic ovaries contain more visible follicles than the average ovary } \\
\hline \multicolumn{4}{|l|}{ Polycystic ovaries tend to ovulate less frequently than the average ovary } \\
\hline \multicolumn{4}{|l|}{ Ovulating more frequently will improve my fertility } \\
\hline \multicolumn{4}{|l|}{$\begin{array}{l}\text { After egg release (ovulation), the hormone progesterone is released; progesterone would allow my } \\
\text { uterine lining to shed and allow me to have a normal menstrual period }\end{array}$} \\
\hline \multicolumn{4}{|l|}{$\begin{array}{l}\text { Having monthly increases in progesterone, and therefore menstrual periods, would decrease my risk } \\
\text { for cancer of the uterus }\end{array}$} \\
\hline \multicolumn{4}{|l|}{ Polycystic ovaries tend to make more male hormone like (testosterone) than the average ovary } \\
\hline \multicolumn{4}{|l|}{ The amount of fat in the body affects the amount of free testosterone in my body } \\
\hline \multicolumn{4}{|l|}{ More free testosterone promotes unwanted hair growth, acne, and scalp hair loss (alopecia) } \\
\hline \multicolumn{4}{|l|}{$\begin{array}{l}\text { Medications that stop the production of male hormone by the ovary (like hormonal contraception) or } \\
\text { block the effects of male hormone would help with unwanted hair growth, acne, and alopecia }\end{array}$} \\
\hline \multicolumn{4}{|l|}{ Insulin helps the ovary to make more male hormone } \\
\hline \multicolumn{4}{|l|}{ High insulin levels are common in women with PCOS } \\
\hline \multicolumn{4}{|l|}{ High insulin levels place individuals at risk for diabetes } \\
\hline \multicolumn{4}{|l|}{$\begin{array}{l}\text { Lowering insulin levels may help decrease free testosterone, help with weight loss and help trigger } \\
\text { ovulation }\end{array}$} \\
\hline \multicolumn{4}{|l|}{ Insulin levels can be lowered by exercise and by certain changes in diet } \\
\hline \multicolumn{4}{|l|}{$\begin{array}{l}\text { Insulin levels can be lowered by medications that make the body more sensitive to insulin, like } \\
\text { metformin }\end{array}$} \\
\hline \multicolumn{4}{|l|}{$\begin{array}{l}\text { Increasing the amount of muscle in my body will increase my body's metabolic rate, that is my } \\
\text { body's ability to burn calories }\end{array}$} \\
\hline \multicolumn{4}{|l|}{$\begin{array}{l}\text { The metabolic syndrome is a group of symptoms that increases my risk for diabetes and } \\
\text { cardiovascular disease }\end{array}$} \\
\hline \multicolumn{4}{|l|}{ Metabolic syndrome is common in women with PCOS } \\
\hline \multicolumn{4}{|l|}{ Exercise, healthy dieting and weight loss can decrease the risk of having metabolic syndrome } \\
\hline Enough about PCOS to explain what it is, and how it affects my health & & & \\
\hline
\end{tabular}

